\title{
L'EOAD en Europe, mythe et réalités
}

Open and Distance Education in Europe, myth and realities

La educación abierta y a distancia, mito y realidades

\section{Serge Pouts-Lajus et Murielle Riché-Magnier}

\section{OpenEdition}

\section{Journals}

Édition électronique

URL : http://journals.openedition.org/ries/2717

DOI : $10.4000 /$ ries. 2717

ISSN : 2261-4265

Éditeur

Centre international d'études pédagogiques

Édition imprimée

Date de publication : 23 septembre 1999

Pagination : 19-28

ISSN : 1254-4590

Référence électronique

Serge Pouts-Lajus et Murielle Riché-Magnier, "L'EOAD en Europe, mythe et réalités », Revue internationale d'éducation de Sèvres [En ligne], 23 | 1999, mis en ligne le 23 septembre 2002, consulté le 03 mai 2019. URL : http://journals.openedition.org/ries/2717 ; DOI : 10.4000/ries.2717 


\section{L'EOAD en Europe, mythe et réalités}

Serge Pouts-Lajus,

Marielle Riché-Magnier ${ }^{1}$

\section{Open and Distance Education in Europe, myth and realities}

Is Open and Distance Education the right solution for European education systems? The authors wonder whether American and European systems are compared too often. They insist that the development of Open and Distance Education in Europe is threatened by approximation and bad valuations.

\section{La educación abierta y a distancia, mito y realidades}

¿Es la educación abierta y a distancia la verdadera solución para los sistemas educativos europeos? Los autores se preguntan si una comparación abusiva con la situación norteamericana es buena. Subrayan las aproximaciones y errores de apreciación que amenazan el desarrollo de la educación abierta y a distancia.

On entend souvent dire, et cela depuis plus de dix ans, que l'éducation et la formation ouvertes et à distance (EOAD) sont promises à une croissance rapide. Pour appuyer cette allégation, des facteurs culturels, sociologiques, économiques, politiques et industriels sont tour à tour invoqués. Pour faire bref, aujourd'hui, les Européens attendraient de leurs institutions éducatives qu'elles leur offrent un service individualisé, adapté à leurs besoins pédagogiques du moment, un enseignement du "juste à temps " faisant fi des contraintes spatiales et temporelles. Face à cette demande, les universités européennes devraient s'attendre à subir la concurrence croissante de leurs homologues américaines, australiennes, asiatiques dont les activités sur ce nouveau front de l'industrie culturelle prennent une ampleur nouvelle à la faveur du développement de l'internet et des réseaux de communication.

1 Serge Pouts-Lajus et Marielle Riché-Magnier ont co-écrit L'Ecole à l'heure d'internet - Les Enjeux du multimédia dans l'éducation, Nathan, 1998. Les opinions exprimées dans cet article n'engagent que leurs auteurs et ne reflètent pas nécessairement la position de la Commission européenne. 


\section{L'EOAD et la société de l'information}

L'individualisation croissante des comportements et des valeurs est l'un des traits marquants de nos sociétés. L'affaiblissement des réseaux de socialisation traditionnels, privés et publics, la famille élargie, la communauté villageoise ou religieuse ou encore les corporations professionnelles, et, en contrepoint, l'exaltation de l'individu, de sa réussite personnelle, en sont les signes les plus visibles. Dans ce contexte, l'éducation tend à devenir une affaire privée qu'il appartiendrait à chacun de gérer de façon autonome, "tout au long de sa vie " ${ }^{2}$. Les institutions d'éducation et de formation s'interrogent sur les nouvelles formes que pourrait emprunter leur offre pour mieux l'adapter à la demande, telle qu'elles la perçoivent ; un nouveau vocabulaire apparaît pour la qualifier, avec tous les panachages possibles : différenciée, flexible, ouverte, à distance, à la carte, sur mesure, voire tout récemment, virtuelle ou encore duale.

Cette tendance est également renforcée par l'évolution de nos économies : les structures industrielles sont profondément affectées par l'importance croissante de la gestion de "l'immatériel " au sein des activités productives. L'ouverture des marchés et la globalisation des échanges favorisent les entreprises, grandes ou petites, capables d'innover, d'évoluer avec le marché, de se positionner rapidement sur des créneaux porteurs, de faire preuve d'une grande flexibilité dans leur organisation. Les progrès scientifiques et technologiques sont tels que les savoirs acquis lors de la formation initiale doivent, dans certains secteurs, être souvent actualisés. Une étude récente menée aux États-Unis évalue à plus de $30 \%$ l'accroissement de la demande individuelle d'éducation au cours des dix prochaines années.

Mais comment financer cette demande croissante d'éducation et de formation dans une période marquée par une certaine rigueur budgétaire ? N’y aurait-il pas lieu d'augmenter l'efficacité de l'enseignement tout en rationalisant ses facteurs de production, voire d'envisager de confier au secteur privé une part plus grande de responsabilité dans l'offre de ce service ? L'EOAD est l'un des moyens avancés de façon récurrente pour relever ce défi. Lui seul paraît être en mesure d'apporter à chaque citoyen, adulte en reconversion, chômeur, personne isolée ou handicapée, un accès à des ressources éducatives qui lui permettront de se former sans contrainte, librement et à son rythme, et cela à un coût supportable pour lui-même et pour la collectivité.

Signes annonciateurs de ces temps nouveaux, des universités et des centres de formation nord-américains utilisent le réseau de l'internet pour promouvoir et diffuser leur offre d'enseignement à distance dans le monde entier.

2 Expression directement traduite du Life Long Learning des anglophones. 
Les fournisseurs européens, cantonnés à des publics de proximité, ignorants pour la plupart d'entre eux des dures lois de la compétition commerciale, paraissent bien mal préparés pour affronter cette nouvelle concurrence.

Dernier facteur : la diffusion des technologies d'information et de communication dans la société, la baisse du prix des télécommunications, l'augmentation des performances techniques des réseaux, la disponibilité d'une vaste palette d'outils dérivés de l'informatique, la multiplication enfin des recherches sur l'EOAD et des expérimentations dans l'enseignement supérieur et la formation professionnelle préparent, depuis plus de dix ans, le terrain d'une mutation globale de l'offre.

L'EOAD va-t-elle donc finir par s'imposer?

\section{Un concept flou, une réalité mal connue}

Avant de confronter, le temps de ces quelques lignes, l'image un peu abstraite de l'EOAD et de ses promesses avec la réalité des pratiques et des expériences, il nous semble utile de nous interroger sur un concept dont le moins que l'on puisse dire est qu'il est flou et que ses applications concrètes le sont davantage encore.

L'histoire peut nous y aider. L'EOAD s'est d'abord construite à partir d'une pratique, celle de l'enseignement par correspondance qui, dès le milieu du XIXe siècle en Angleterre et à la fin du siècle en France, pourvoyait aux besoins éducatifs de personnes empêchées de participer, pour des raisons professionnelles ou physiques, à des cours collectifs. L'idée de « distance » est bien, à l'origine, celle d'une distance spatiale et temporelle, la prestation du service étant marquée par le temps d'acheminement du courrier entre l'élève et son tuteur. L'enseignement par correspondance, dont l'objectif principal est d'élargir l'audience des services d'éducation et de formation à des publics nouveaux, porte en germe la notion d'adaptation aux besoins individuels par l'ouverture et la flexibilité.

La généalogie de l'EOAD est également marquée par le développement des technologies d'information et de communication qui ont été parallèlement expérimentées dans les deux dispositifs éducatifs, présentiel et à distance. Dans un premier temps, cette évolution a donné un souffle nouveau au concept d'enseignement par correspondance. La radio et la télévision, au tournant du siècle, lors de la deuxième guerre mondiale aux États-Unis, dans les années soixante en Europe et pendant la décolonisation en Afrique, ont été utilisées pour pallier la pénurie de formateurs. Dans les systèmes traditionnels d'éducation initiale et de formation continue, depuis la fin des années soixante-dix, les tentatives d'introduction de logiciels interactifs, malgré de nombreuses difficultés, se sont multipliées. Après quelques essais peu convaincants inspirés des thèses comportemen- 
talistes et de l'enseignement programmé, l'EOAD s'est finalement orientée vers un nouveau " paradigme " centré sur la fonction d'apprentissage et la relation pédagogique entre l'élève et son tuteur. Cette vision qui rencontre un succès croissant conduira, dans un second temps, le courant technologique à investir totalement l'enseignement par correspondance dont le nom même finit par disparaître du paysage ; au milieu des années quatre-vingt en France, le Centre national d'enseignement par correspondance devient le Centre national d'enseignement à distance ; les universités qui se créent pour délivrer des cours par correspondance prennent désormais le nom d'universités ouvertes, d'universités à distance ou plus joliment, au Japon, d'université de l'air.

La piste historique se brouille depuis quelques années sous l'effet de la diversification des pratiques au sein de l'enseignement à distance comme du présentiel. A côté du tutorat à distance traditionnel, les institutions qui se réclament de l'EOAD mettent en place des sessions en "face à face " afin de rompre l'isolement et de favoriser des échanges pédagogiques directs au sein de groupes d'apprenants. A l'inverse, dans le cadre présentiel, scolaire ou universitaire, on voit se développer des sessions à distance par vidéoconférences ou sur l'internet, complémentaires de l'enseignement traditionnel. La visioconférence est par exemple exploitée par les lycées de certaines académies pour élargir l'accès à des disciplines ou à des options rares qui ne peuvent être enseignées dans tous les établissements, ou pour expérimenter des modes d'enseignement mutuel. En France toujours, des universités regroupées au sein du RUCA (réseau universitaire des centres d'autoformation) ont produit un ensemble cohérent de ressources pédagogiques sur un site web destinées aux étudiants de premier cycle et qui peuvent être exploitées " pour illustrer ou compléter l'enseignement traditionnel, pour une autoformation en salles de ressources ou en bibliothèques ou encore, hors de l'établissement, pour un enseignement à distance, dans un contexte d'apprentissage en autonomie accompagnée ${ }^{3}$.

Un même flou conceptuel se retrouve dans la recherche sur l'EOAD. Pour certains chercheurs, cette forme d'enseignement se signale par la simple médiation des technologies; pour d'autres, elle relève essentiellement d'une approche pédagogique originale faisant une large part à la flexibilité. Position ultime, celle revendiquée par l'université française dont la thèse officielle repose sur l'idée d'une " convergence entre l'enseignement présentiel et l'enseignement à distance », c'est-à-dire sur l'absorption de l'un par l'autre ${ }^{4}$. Ecartelé entre dif-

3 Ces ressources sont consultables à l'adresse : « http://www.univ-lillel.fr/lemm/ruca/pcsm.htm ».

4 Voir le site " http://www.educnet.education.fr/doc/pays/boffrefor.htm " du ministère de l'Education nationale ; cette même idée de convergence est présente dans le rapport du député Patrick Bloche : Le désir de France - La présence internationale de la France et de la francophonie dans la société de l'information, Rapport au premier ministre, 7 décembre 1998, consultable sur " http://www.internet.gouv.fr/francais/textesref/rapbloche98/sommaire.htm . 
férentes écoles, le concept perd à la fois de sa force et de sa substance. Dès lors que la réalité de l'EOAD est mal cernée, que la notion et les enjeux sont mal définis, tous les espoirs et toutes les craintes sont permis. Mais depuis plus de dix ans que la percée de l'EOAD et la diffusion internationale de ce mode d'enseignement sont annoncées comme imminentes, ne serait-il pas utile de faire un arrêt sur image et d'aller sur le terrain en constater la réalité et la forme ? La tentation de réduire l'EOAD à une idée ou, mieux, à une utopie ne fait peut-être que refléter la difficulté d'appréhender une pratique en mouvement.

\section{Les stratégies institutionnelles}

L'effet de brouillage constaté dans la construction du concept et dans les directions prises par la recherche se manifeste également dans des stratégies institutionnelles qui paraissent hésitantes, parfois contradictoires. Dans l'enseignement supérieur, les problématiques de l'EOAD se sont en partie cristallisées sur la question de l'université ouverte. Au cours des trente dernières années, cinq pays de l'Union européenne ont choisi de créer une structure nationale dédiée à la diffusion d'un enseignement universitaire à distance : l'Allemagne, les PaysBas, le Portugal, l'Espagne ${ }^{5}$ et le Royaume-Uni. Une majorité de neuf pays ${ }^{6}$, confrontés à cette question, n'ont pas opté pour la création d'une institution spécialisée.

Comment interpréter ce constat ? L'hypothèse d'une avant-garde explorant des voies innovantes devant un groupe de pays encore hésitants ne peut ici être retenue. Les cinq universités ouvertes nationales existantes ont été fondées entre 1970 et $1987^{`}$. Or, depuis cette date, la question de la création de structures d'enseignement supérieur à distance a en permanence été débattue dans l'ensemble des pays de l'Union européenne. Des groupes de pression très actifs ont milité sans succès pour leur création dans les pays où elles n'existent pas. En réalité, le développement de l'offre d'enseignement supérieur et de formation professionnelle à distance emprunte principalement deux voies : l'une consiste à concentrer cette offre sur une institution spécialisée, une université ouverte, l'autre à la diffuser en s'appuyant sur le tissu universitaire existant. La première voie est privilégiée par des pays comme le Royaume-Uni, dont le réseau universitaire est peu dense ; la seconde convient bien aux pays comme la France, qui se

5 L'Espagne est le seul pays d'Europe ayant deux universités ouvertes, l'une nationale (UNED), l'autre régionale (Catalogne).

6 Le Luxembourg, pour raison de taille, n'est pas concerné.

7 Royaume-Uni (Open University) : 1970 ; Espagne (Universidad Nacional de Educación a Distancia) : 1972 ;

Allemagne (Fern Universität) :1975 ; Pays-Bas (Open Universiteit) : 1984 ; Portugal (Universidade Aberta) : 1987. 
trouvent dans une situation inverse et préfèrent logiquement étendre les pratiques d'enseignement ouvert à l'ensemble du tissu plutôt que de les concentrer sur une structure unique ${ }^{8}$. La solution originale mise en place en Italie mérite d'être mentionnée ; plusieurs universités se sont associées dans deux structures de mutualisation des moyens : le Consorzio per l'Università a Distanza et le Consorzio Nettuno. Parmi les raisons qui plaident contre la création d'établissements spécialisés, en dehors des structures universitaires, il faut également signaler le risque rarement évoqué mais pourtant bien réel de dévalorisation des diplômes délivrés par ces établissements.

Dans tous les pays européens, qu'ils soient ou non dotés d'une université ouverte, chaque établissement d'enseignement supérieur est confronté à la question de l'EOAD. C'est donc aussi à ce niveau que l'on peut tenter de cerner l'orientation des stratégies et des pratiques. Or, il est frappant de constater que l'on retrouve ici la même alternative entre une stratégie de « concentration " dans une entité spécialisée et celle de "diffusion » dans l'ensemble des composantes d'un établissement. Les observations dont nous disposons montrent que dans les universités européennes, les stratégies de concentration sont généralement dominantes. Elles prennent deux formes. La première consiste à créer des structures spécialisées, équipées du matériel technologique adéquat, et chargées de mettre sur pied une offre d'EOAD en coopération avec des unités pédagogiques volontaires. En France, on dénombre vingt-cinq centres de télé-enseignement universitaires ${ }^{9}$ fonctionnant sur ce mode. La deuxième forme de concentration des problématiques d'EOAD dans une université, la plus répandue, consiste à confier aux départements de sciences de l'éducation ou de formation des enseignants la responsabilité de la recherche et de l'expérimentation en EOAD. Lorsque l'on s'avise de repérer concrètement les pratiques d'EOAD dans une université, on se retrouve très souvent face à des étudiants qui sont eux-mêmes de futurs enseignants ou de futurs chercheurs dans le domaine des sciences de l'éducation, et qui expérimentent cette pratique dans le cadre d'un cours dont le sujet est précisément l'EOAD.

La stratégie de concentration, qu'on la considère au niveau d'un pays ou d'un établissement, présente quelques avantages mais un inconvénient très sérieux : certes, elle engendre à court terme du dynamisme et de la visibilité mais en même temps, elle provoque des effets pervers de démobilisation des unités non concernées ${ }^{10}$. Pour la même raison qui fait que la création d'une université ouverte n'est pas forcément la meilleure stratégie pour un pays, la créa-

8 En France, la promotion du puissant CNED en université ouverte nationale, souvent envisagée, ne s'est pas faite et, à notre connaissance, n'est plus à l'ordre du jour.

9 Leur liste peut être consultée à l'adresse : " http://telesup.univ-mrs.fr/ListeCTEU.html ".

10 Des effets du même ordre sont bien connus dans le monde de l'entreprise : si l'on veut que l'innovation, la qualité ou les nouvelles technologies soient l'affaire de tous, mieux vaut éviter de créer des entités trop puissantes dont ce serait le champ unique. 
tion d'un département de télé-enseignement peut ne pas l'être pour une université ${ }^{11}$.

\section{L'exception européenne}

La comparaison avec les États-Unis sert souvent de référence pour justifier l'inéluctable poussée de l'EOAD en Europe. C'est ignorer qu'aux ÉtatsUnis, l'enseignement supérieur fonctionne comme un marché très concurrentiel, ce qui est loin d'être le cas en Europe ; les Européens peuvent n'avoir ni besoin ni envie d'imiter les stratégies commerciales d'universités américaines de renommée internationale (Stanford, Yale, Harvard, etc.) qui cherchent à vendre leurs services au-delà de leurs frontières. Penser que nous devrions suivre la voie tracée par les États-Unis, c'est surtout confondre la mise en ligne de cours sur le web avec une évolution pédagogique, alors que dans la plupart des cas, il ne s'agit que d'une activité assez classique d'exploitation de produits dérivés qui ne préjuge en rien de leur usage. Il est par ailleurs important de ne pas confondre un phénomène limité à quelques universités avec la question plus générale dont il est question ici, et qui porte sur l'évolution des méthodes d'apprentissage et de diffusion des services d'enseignement.

$\mathrm{Au}$-delà des raisons géographiques qui expliquent le recours à l'EOAD dans des pays comme l'Australie, le Japon et ses îles, les États-Unis ou la Finlande en Europe, comment ne pas imaginer que, dans un domaine aussi dépendant de l'histoire, des cultures et des traditions que l'enseignement, ces différences ne portent pas également sur l'organisation des services éducatifs et sur les méthodes pédagogiques?

Sans s'attarder sur les raisons, nombreuses, qui concourent à rendre l'EOAD moins attrayante pour les Européens que pour les Américains, il paraît important de rappeler l'importance de la dimension sociale de l'éducation sur le vieux continent, moins développée outre-Atlantique. L'éducation et la formation sont ici un service public avant tout, un droit acquis par le citoyen, et là davantage un marché. Elles sont ici l'affaire de la société, des institutions et des entreprises ${ }^{12}$, et là davantage celle de l'individu, principal responsable de son itinéraire professionnel. L'EOAD se heurte également dans certains pays à des perceptions bien ancrées de l'éducation, du métier d'enseignant, des méthodes pédago-

11 Le rapport Restructurer l'université - Les nouvelles technologies dans l'enseignement et l'apprentissage - Pistes stratégiques pour l'université du Conseil des recteurs francophones, avril 1998, souligne les interrogations, les difficultés mais aussi l'absence de stratégies institutionnelles claires des universités tant européennes que nord-américaines face à l'introduction des technologies dans l'enseignement.

12 En France, rappelons-le, les entreprises sont contraintes par la loi de consacrer au moins 1,1\% de leur masse salariale à la formation continue des salariés. 
giques : si le tutorat, mode d'enseignement emblématique de l'EOAD, semble bien accepté dans les pays anglo-saxons tant par les enseignants que par les élèves, les cultures latines valorisent davantage le dialogue direct et la présence physique d'un enseignant face à un groupe d'élèves partageant les mêmes objectifs d'apprentissage. Dès lors que l'on s'interdit toute comparaison qualitative qui impliquerait, par exemple, de qualifier certains traits culturels de conservateurs et d'autres de progressistes, il n'y a aucune raison d'interpréter le développement de l'EOAD comme un facteur de progrès en lui-même.

Quelles que soient la culture et la tradition, l'enseignement à distance nous semble de façon générale mal adapté au public scolaire. Les enfants ont autant besoin d'apprendre à vivre dans la micro-société que constitue l'école que d'apprendre à lire, écrire et compter ; les études universitaires commencent à un âge qui s'accompagne souvent d'un fort désir de sociabilité et de rupture avec le milieu familial ainsi qu'avec les méthodes pédagogiques de l'enseignement secondaire. Or, pour réussir, l'EOAD doit absolument échapper à toute assimilation avec un pis-aller, misérable, dégradé ou régressif ${ }^{13}$.

\section{Du concept aux pratiques}

Les contextes nationaux, les modèles sociaux et les attentes individuelles sont donc les éléments déterminants du développement et de l'orientation des pratiques d'EOAD. En Europe, quoi que l'on dise, l'EOAD demeure embryonnaire et principalement expérimentale. On peut bien sûr multiplier les exemples prometteurs mais limités à des publics présentant des besoins très particuliers comme, sur le plan scolaire, les enfants hospitalisés ${ }^{14}$, les enfants de forains ${ }^{15}$, ou encore les jeunes sportifs de haut niveau tels que les " cyberchampions " du plateau de Villard-de-Lans ${ }^{16}$. L'EOAD est le plus souvent expérimentée dans des contextes exceptionnellement favorables, comme par exemple dans le cadre du projet Learn-Nett ${ }^{17}$ auquel collaborent cinq universités belges et leurs étudiants des départements d'éducation et de technologies de l'éducation ; la même observation s'applique aux expérimentations d'EOAD pour la formation continue des

13 De nombreuses études montrent que le taux d'abandon de cours à distance en Europe comme aux États-Unis se situe entre 30 à $40 \%$ des inscrits, essentiellement pour des raisons de motivation.

14 Voir par exemple l'activité de l'association «L'enfant à l'hôpital» qui intervient dans une soixantaine d'hôpitaux français et belges.

15 Voir le projet de recherche européen Topilot dont les résultats peuvent être consultés sur le site de la Commission européenne à l'adresse : " http://www2.echo.lu/telematics/education/ ".

16 Expérience qui concerne des lycéens appelés à quitter cinq mois durant leur établissement pour participer à des compétitions de ski.

17 Voir à l'adresse : « http://www.ulb.ac.be/project/learnet/ ". 
ingénieurs d'IBM ou de France Télécom ${ }^{18}$. Les nombreuses expériences et recherches menées dans ces conditions par des universités et des centres de formation, souvent dans le cadre de programmes européens, ne permettent généralement pas de conclure que les modèles appliqués aient quelque chance de pouvoir être étendus avec succès à des groupes plus larges et moins favorablement disposés. Dans de nombreux cas, les résultats des expériences réussies se résument à des publications dans des revues spécialisées et au montage d'une nouvelle expérience bien souvent destinée à tester un outil technique entre-temps apparu.

Ce constat quelque peu pessimiste ne doit cependant pas masquer certaines réussites. Par exemple, celles que l'on rencontre dans la formation continue et professionnelle lorsque, reprenant le flambeau et l'esprit de l'enseignement par correspondance et de la promotion sociale, des organismes de formation aguerris permettent à des adultes motivés de compléter, après le travail, leurs qualifications ou d'en acquérir de nouvelles ${ }^{19}$. Ces pratiques rappellent que les approches " par le bas ", qui privilégient la prise en compte des particularités du contexte et de la demande réelle, sont peut-être plus efficaces que les approches " par le haut » qui cherchent à appliquer des concepts et des modèles généraux. L'EOAD s'avère utile lorsqu'elle est nécessaire, lorsqu'existe un public pour qui l'accès à des enseignements présentiels est rendu difficile ou partiellement impossible. Ce public se décline en autant de situations sociales, de classes d'âges, de besoins pédagogiques spécifiques, de caractéristiques culturelles qui ne peuvent être négligés.

Le développement de l'EOAD en Europe est menacé par deux erreurs d'appréciation, toutes deux ressortissant à la même tentation d'approcher le problème par le haut. La première se concentre sur la mobilisation des autorités politiques et administratives ; en voulant à tout prix les convaincre de l'existence d'un avantage spécifique de l'EOAD sur les modalités plus traditionnelles, ses promoteurs courent le risque d'être démentis par les faits, incapables qu'ils seront d'apporter la preuve effective que « l'EOAD fait la différence ${ }^{20}$. La seconde se focalise sur la technologie. En cédant à la tentation d'entrer dans la problématique de l'EOAD par la seule porte technologique, on ne fait que réduire, sur le plan de l'ingénierie éducative, le champ des possibles. Des décennies de recherche en sciences de l'éducation le montrent pourtant bien : l'efficacité d'un dispositif d'apprentissage ne peut pas se mesurer à l'aune des outils et des

18 Pour d'autres exemples en Europe, voir l'étude financée par le programme européen Socrates : Technology Enhanced Evaluation in Open and Distance Learning (TEEODE), Bartolomé Pina \& Underwood, 1998.

19 Voir par exemple les formations assurées par le CNAM des pays de Loire : " http://www.cnampaysdelaloire.fr/pagescadres/cadres3.htm ".

20 C'est dans ce sens que doit être lu le rapport What's the Difference, publié aux États-Unis par the Institute for Higher Education Policy en avril 1999, qui épingle les fautes méthodologiques de la plupart des recherches visant à démontrer l'efficacité de l'EOAD. 
méthodes qu'il emploie. C'est au contraire en payant pleinement tribut aux particularismes des demandes et des solutions que l'on pourra espérer dégager peu à peu des enseignements exemplaires et envisager, sur cette base, le transfert d'expérience. 